\title{
Comparative Measures of Single-Wall Carbon Nanotube Dispersion
}

\author{
J. A. Fagan, ${ }^{*} \uparrow$ B. J. Landi, ${ }^{\S}$ I. Mandelbaum, ${ }^{\ddagger}$ J. R. Simpson, ${ }^{\ddagger}$ V. Bajpai, ${ }^{\dagger}$ B. J. Bauer, ${ }^{\dagger}$ \\ K. Migler, ${ }^{\dagger}$ A. R. Hight Walker, ${ }^{\ddagger}$ R. Raffaelle, ${ }^{\S}$ and E. K. Hobbie ${ }^{*, \dagger}$ \\ Polymers Division, National Institute of Standards and Technology, Gaithersburg, Maryland 20899, \\ Physics Laboratory, National Institute of Standards and Technology, Gaithersburg, Maryland 20899, and \\ NanoPower Research Laboratories, Rochester Institute of Technology, Rochester, New York 14623
}

Received: July 25, 2006; In Final Form: September 14, 2006

\begin{abstract}
Model composites of DNA-wrapped single-wall carbon nanotubes in poly(acrylic acid) are used to evaluate metrics of nanotube dispersion. By varying the $\mathrm{pH}$ of the precursor solutions, we introduce a controlled deviation from ideal behavior. On the basis of small-angle neutron scattering, changes in near-infrared fluorescence intensity are strongly correlated with dispersion, while optical absorption spectroscopy and resonant Raman scattering are less definitive. Our results represent the first systematic comparison of currently accepted measures of nanotube dispersion.
\end{abstract}

\section{Introduction}

Single-wall carbon nanotubes (SWNTs) exhibit remarkable physical properties and have the potential for profound impact over a broad range of technologies, from first-generation applications such as conducting and mechanically reinforced plastics to more advanced applications such as fuel cells, microelectronic components, flat panel displays, and biochemical sensors. ${ }^{1}$ In many of these applications, efficient dispersion of SWNTs at the individual nanotube level will be a prerequisite for optimal performance. Sources of imperfection in dispersing individual SWNTs are most likely bundles, defined here as the lengthwise "roping" of tubes, and aggregates, the fractal-type networking of tubes in a floc. Of all the measurement techniques currently used to evaluate SWNT dispersion in solutions and composites, small-angle scattering ${ }^{2-9}$ is perhaps the simplest to interpret and understand. This technique directly probes twopoint correlations in composition and can thus distinguish true form scattering due to individual SWNTs from the structural scattering that arises from nanotube aggregates and bundles. Other more readily available methods, however, have also recently been used to evaluate SWNT dispersion, typically by focusing on subtle effects such as those that tube-tube interactions have on the optical signatures associated with transitions between electronic states in SWNTs of different chirality. Most notably, these techniques include optical absorption spectroscopy, ${ }^{10}$ near-infrared (NIR) fluorescence spectroscopy, ${ }^{1-14}$ and resonant Raman scattering. ${ }^{15-19}$ To extract maximum information from these optical techniques, the sensitivity of each to the level of SWNT dispersion must be known, especially as the limit of individually dispersed tubes is approached.

Despite claims of SWNT dispersion throughout the literature, there is currently no consensus on an absolute measure, nor has there been a methodical comparison of different approaches that might serve as a foundation for such a measure. In this

* Corresponding authors. E-mail: (Fagan) jeffrey.fagan@nist.gov; (Hobbie) erik.hobbie@nist.gov.

Polymers Division, National Institute of Standards and Technology.

$\doteqdot$ Physics Laboratory, National Institute of Standards and Technology.

$\S$ NanoPower Research Laboratories, Rochester Institute of Technology. paper, model polymer composites composed of single-stranded deoxyribonucleic acid (ssDNA)-stabilized SWNTs dispersed in poly(acrylic acid) (PAA) are used for an evaluation of the different metrologies currently used to assess SWNT dispersion. By varying the $\mathrm{pH}$ of the precursor PAA-SWNT solution, a systematic variation in SWNT aggregate dispersion is evaluated by scattering and optical spectroscopy. Small-angle neutron scattering (SANS) provides the most direct measure of dispersion. Near-infrared fluorescence spectroscopy, proposed to be sensitive to the bundling or clustering of SWNTs, similarly exhibits a definitive measure. In contrast, optical absorption spectroscopy and resonant Raman scattering have limited sensitivity for dispersions without appreciable bundling. Our study represents the first controlled comparison of currently accepted metrics for evaluating SWNT dispersion and serves as a useful point of reference for any of the four techniques.

\section{Experimental Section}

Aqueous dispersion of SWNTs ${ }^{20}$ was achieved by sonication (0.32-cm tip sonicator, Thomas Scientific) of HiPco SWNTs (Carbon Nanotechnologies Inc. Batch P025721) in salt solution ( $200 \mathrm{mmol} / \mathrm{L} \mathrm{NaCl}, 100 \mathrm{mmol} / \mathrm{L}$ Tris, $5 \mathrm{mmol} / \mathrm{L} \mathrm{NaN}$ buffered to $\mathrm{pH} 7$ with $\mathrm{HCl}$ ) in the presence of 30 -mer $5^{\prime}-\mathrm{GT}(\mathrm{GT})_{13} \mathrm{GT}$ $3^{\prime}$ single-stranded DNA (Integrated DNA Technologies ${ }^{21}$ ). SWNT powder and DNA were both loaded at $1 \mathrm{mg} / \mathrm{mL}$. In all cases, the sonication was performed in a $15-\mathrm{mL}$ centrifuge tube immersed in an ice bath and tightly covered to reduce evaporation. The sonication period was $2 \mathrm{~h}$ at $9 \mathrm{~W}$ of applied power. Post-sonication suspensions were further processed by centrifugation at $21000 \mathrm{~g}$ in $1.5-\mathrm{mL}$ centrifuge tubes for $2 \mathrm{~h}$; the resulting supernatant is a stable, rich black liquid containing well-dispersed SWNT material. ${ }^{20,22}$ PAA-SWNT composites were prepared by mixing the aqueous DNA-stabilized SWNT dispersion with a $25 \%$ by mass solution of PAA in water $(240 \mathrm{k}$ MW, Sigma-Aldrich \#19205-8, ${ }^{21}$ used as received) at a mixing ratio of 1 to 4 by mass. SWNT-free mixtures of PAA and the corresponding salt buffer were also processed in the same manner. Alteration of the $\mathrm{pH}$ of the PAA was performed, when specified, prior to mixing with the SWNT dispersion by the addition of $\mathrm{NaOH}$ to the aqueous PAA solution. The PAA- 
SWNT solutions were vigorously mixed to ensure uniformity and were subsequently allowed to stabilize for at least $24 \mathrm{~h}$ before casting; this time allows for the dissolution or creaming of bubbles from the solution so that defects are not cast into the composite. Rested solutions were cast on a poly(dimethylsiloxane) (PDMS) surface and allowed to dry in air for approximately 1 day; polymer composites of PAA are peeled easily from the casting surface once nominally dry and were subsequently desiccated under vacuum. Two specific pairs of composite samples were prepared by this method on the basis of the acidity of their initial PAA-SWNT solutions and used for all of the measurements; these are denoted as "unbuffered" (pH 2.2) and "buffered" ( $\mathrm{pH} 4.1)$.

For atomic force microscopy (AFM) measurements only, sample preparation involved dialysis of a portion of the aqueous ssDNA/SWNT suspension through a 50000 relative molecular mass membrane (Spectra-Por ${ }^{21}$ ) against $\mathrm{pH} 7$ distilled water. Freshly cleaved mica surfaces were dipped into the dialyzed solution for $5 \mathrm{~s}$, rinsed with distilled deionized water, and finally dried in an oven at $50{ }^{\circ} \mathrm{C}$ for $5-10 \mathrm{~min}$. The dialysis step was performed to reduce the amount of salt deposited during sample preparation. Images of the SWNTs on the prepared surfaces were obtained in air using a Digital Instruments NanoScope IV. ${ }^{21}$

Small-angle neutron scattering (SANS) was performed on the 30-m NG7 instrument at the National Institute of Standards and Technology (NIST) Center for Neutron Research (NCNR). Blank samples without SWNTs but with the same chemical and physical history were used to measure background scattering due to the polymer.

UV-vis-NIR spectroscopy was performed in transmission mode on a PerkinElmer Lambda 950 UV-vis-NIR spectrophotometer ${ }^{21}$ over the ranges of $1750-190 \mathrm{~nm}$ (solutions) and 2750-190 nm (composites). In all cases, the incident light was depolarized prior to the sample compartment, and the instrument was corrected for both the dark current and background spectra; data were recorded at 1-nm increments, with an instrument integration time of $0.2 \mathrm{~s}$ per increment. Additionally, the reference beam was left unobstructed, and the subtraction of the appropriate reference sample was performed during data reduction.

Fluorescence maps were generated using a JY-Horiba Fluorolog-3 spectrofluorometer ${ }^{21}$ and corrected for the instrument's source spectral distribution and detector spectral response. Excitation wavelength was scanned in 3-nm increments using a 450-W xenon lamp through a 15-nm slit and a 1200 lines/ $\mathrm{mm}$ monochromator with a 500 blaze. The emission was collected front face and measured using a liquid $\mathrm{N}_{2}$-cooled InGaAs detector over 5-nm increments through a $15-\mathrm{nm}$ slit and a 600 lines/mm monochromator with a 1500 blaze. To obtain a strong NIR fluorescence signal, the composite samples were additionally heated to just above the glass transition temperature of the dry PAA and mechanically stretched to a draw ratio of $4: 1$ to reduce thickness and enhance light transmission. In addition to thinning the film, this degree of stretching orients the SWNTs. As the polymer remains extremely viscous, and the time over which the polymer is heated is less than about $30 \mathrm{~s}$, the SWNTs do not have a chance to otherwise rearrange and stretching has a negligible effect on the dispersion.

The resonant Raman signal was collected in a collinear backscattering configuration. ${ }^{23} \mathrm{~A}$ Ti:sapphire ring laser (Coherent 899 Ring Laser $^{21}$ ) pumped by an Ar-ion laser (Coherent Innova Sabre with multi-line visible head ${ }^{21}$ ) provided a tunable

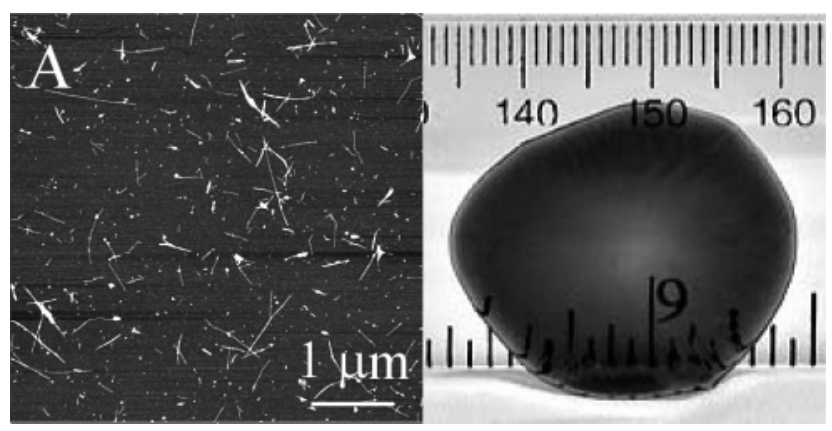

Figure 1. (left) AFM image of ssDNA-wrapped SWNTs from dialyzed stock aqueous suspension and (right) photograph of a fully dried $240 \mathrm{k}$ PAA unbuffered composite of the ssDNA-wrapped SWNTs $(0.035 \%$ SWNT by mass). The AFM image demonstrates the individual dispersion of the DNA-wrapped SWNTs in the original solution; thus, the SWNTs are mixed with the PAA in dispersed form. The composites were optically clear and homogeneous down to $200 \times$ magnification for samples cast from both the buffered $(\mathrm{pH} 4.1)$ and unbuffered $(\mathrm{pH}$ 2.2) PAA solutions.

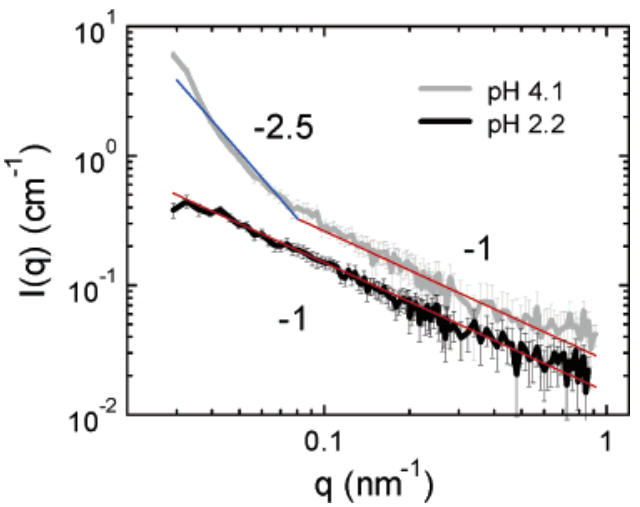

Figure 2. SANS intensity as a function of scattered wave vector for $0.035 \%$ SWNT by mass PAA composites cast from ssDNA-stabilized SWNTs in unbuffered ( $\mathrm{pH} 2.2)$ and buffered $(\mathrm{pH} 4.1)$ aqueous $240 \mathrm{k}$ PAA solution, where the red lines denote a power law with an exponent of -1 and the blue line a power law with exponent -2.5 . The black trace has been shifted down slightly for clarity by a constant multiplication factor of 1.77. Error bars denote two standard deviations in the total experimental uncertainty.

pump source at approximately $10-15 \mathrm{~mW}$ of power focused to a spot size of $10 \mu \mathrm{m}$. The spontaneous Raman backscattered light was collected with a triple-grating spectrometer (f/\# $=6$ aperture, Dilor XY800 ${ }^{21}$ ) and a liquid $\mathrm{N}_{2}$-cooled CCD detector. A linear polarizer was placed before the input aperture to ensure high polarization rejection. The signal was integrated for $(90$ $\pm 0.01) \mathrm{s}$ and averaged over multiple scans. Data were collected over a discrete range of excitation wavelengths in the interval $690 \mathrm{~nm}<\lambda<850 \mathrm{~nm}$ for continuous Raman frequency shifts of $150-400 \mathrm{~cm}^{-1}$, specifically focusing on the radial breathing mode (RBM) of the SWNTs.

\section{Results and Discussion}

Figure 1 shows a typical AFM image of the ssDNA-coated SWNTs obtained from the precursor (no PAA) aqueous suspensions. The preponderance of the deposited SWNTs are individually dispersed on the surface, as was previously shown for DNAwrapped SWNTs in ref 20. This is important as it demonstrates that the SWNTs were initially dispersed prior to the introduction of the PAA. The fully dried SWNT composites were optically transparent and contained no visible clusters down to $200 \times$ magnification (Figure 1), demonstrating macro- to microdispersion; ${ }^{24}$ for differentiating small aggregates and networks 


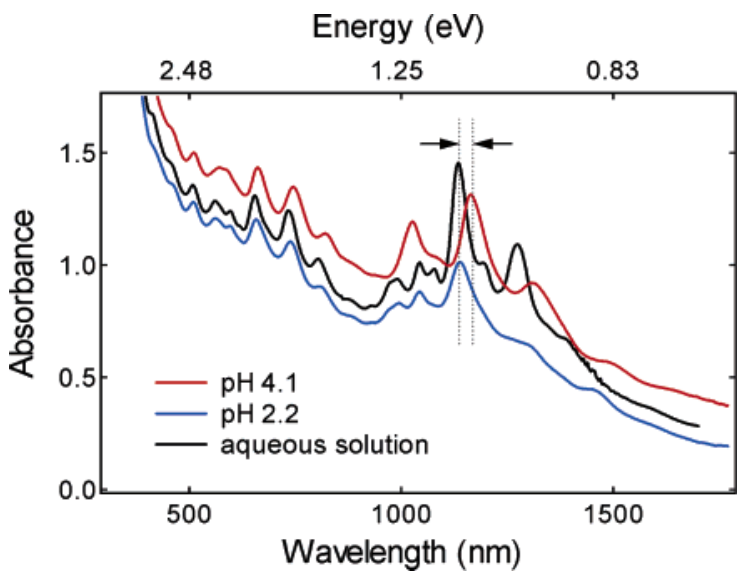

Figure 3. UV-vis-NIR absorbance as a function of wavelength for $0.035 \%$ SWNT by mass PAA composites cast from ssDNA-stabilized

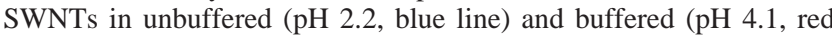
line) aqueous 240k PAA solution, where the solid/black trace denotes the spectra of the same concentration of ssDNA-wrapped SWNTs in pure aqueous suspension ( $\mathrm{pH} 7)$.

from singly "nano-dispersed" material, finer scale measures of SWNT dispersion are required.

SANS profiles from the two composite samples are shown in Figure 2. As stated in the Introduction, SANS offers a potentially powerful method of assessing SWNT dispersion. For an isolated nanotube, the mass contained within a sphere of radius $r$ is $N(r) \propto r^{D}$, where $D$ is the fractal dimension of the tube. The radial pair-distribution function is thus $g(r) \propto$ $r^{1-d}(\delta N / \delta r) \propto r^{D-d}$, where $d=3$ is the spatial dimension. Taking the Fourier transform, the scattering intensity is $I(q) \propto$ $q^{-D}$, where $q$ is the scattered wave vector. For fully dispersed linear objects with $D \approx 1$, one would thus expect $q^{-1}$ behavior. As shown Figure 2, the unbuffered composite exhibits a $q^{-1}$ power law over the entire $q$ range measured, corresponding to rigid-rod behavior over a range of length scales between 6 and
$200 \mathrm{~nm}$. A deviation would be expected at length scales corresponding to the diameter of the SWNT. Since the $q^{-1}$ behavior persists up to $1 \mathrm{~nm}^{-1}$, it was concluded that the diameter of the scattering object is less than $6 \mathrm{~nm}$, consistent with the reported diameters for individual HiPco SWNTs. In comparison, the buffered composite exhibits a distinctive upturn in scattering at low $q$, approximately proportional to $q^{-2.5}$, which is characteristic of nanotube clustering. The difference is both striking and conspicuous, directly demonstrating ideal nanoscale SWNT dispersion in the unbuffered composite, as well as the ability to tune dispersion through adjusting the $\mathrm{pH}$ of the precursor PAA solution. Although we cannot rule out the presence of some small SWNT bundles (diameter less than 6 $\mathrm{nm}$ ) on the basis of SANS alone, resonant Raman scattering (as described in detail below) suggests that the SWNTs in both composites are predominantly debundled and that differences in the SANS profile can thus be attributed to the diffuse aggregation or clustering of individual SWNTs.

Figure 3 shows a comparison of the UV-vis-NIR absorption spectra obtained from the two composites, along with that from the initial aqueous ( $\mathrm{pH} 7$ ) ssDNA-stabilized SWNT suspension. The relative intensity of the peaks corresponding to the excitonic transitions for SWNTs of specific chirality indices $(n, m)$ in the NIR region of the spectrum are reduced from aqueous solution to buffered composite to unbuffered composite, although the unbuffered composite exhibits better resolved spectra than the buffered. The second difference between samples is a red-shift in the position of the dominant absorption peak from $1134 \mathrm{~nm}$ in the aqueous solution and unbuffered composite to $1163 \mathrm{~nm}$ in the buffered composite. To rule out the possibility that this $30-\mathrm{nm}$ shift in peak position is due to $\mathrm{pH}$, absorption measurements on a series of aqueous DNA-SWNT suspensions in which the $\mathrm{pH}$ was adjusted from 7 were performed, over the range $1.6<\mathrm{pH}<10.6$, while maintaining the total salt content at $0.5 \mathrm{~mol} / \mathrm{L}$. Peak shifts associated with the changes to $\mathrm{pH}$ were less than $4 \mathrm{~nm}$, thus indicating that the observed spectral
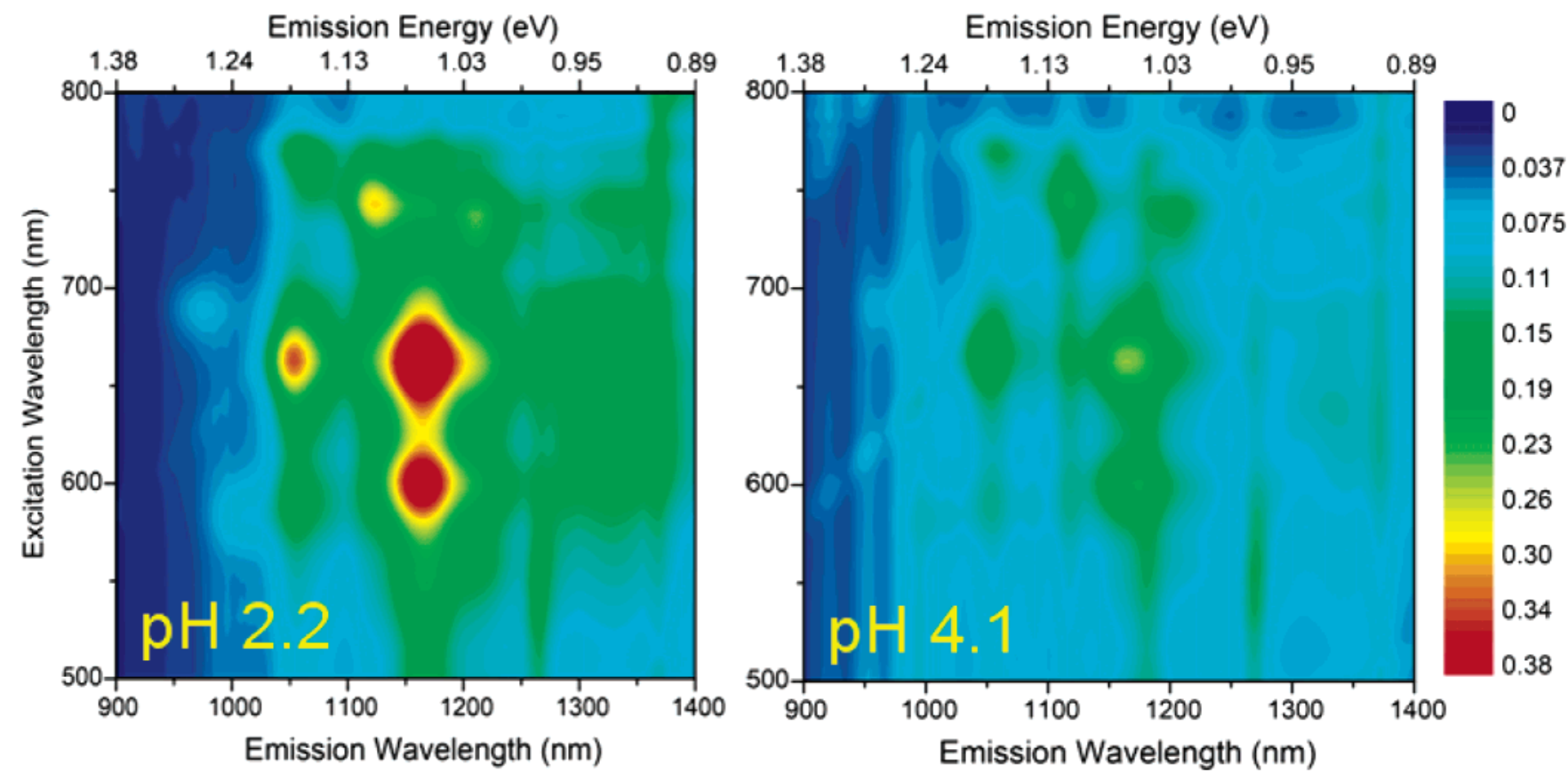

Figure 4. Comparison of NIR fluorescence plots for the SWNT composite samples, where pieces of the identical buffered and unbuffered samples used in all of the measurements were melt-drawn to an aspect ratio of 4 in order to render the films sufficiently transparent. Stretching the polymer films aligns the SWNTs but has no effect on SWNT dispersion. SWNT concentration, film thickness, and draw ratio are the same. The intensity scale is the same for both plots. Despite equal SWNT concentration and near equal film thicknesses, the fluorescence of the unbuffered film is substantially larger. 


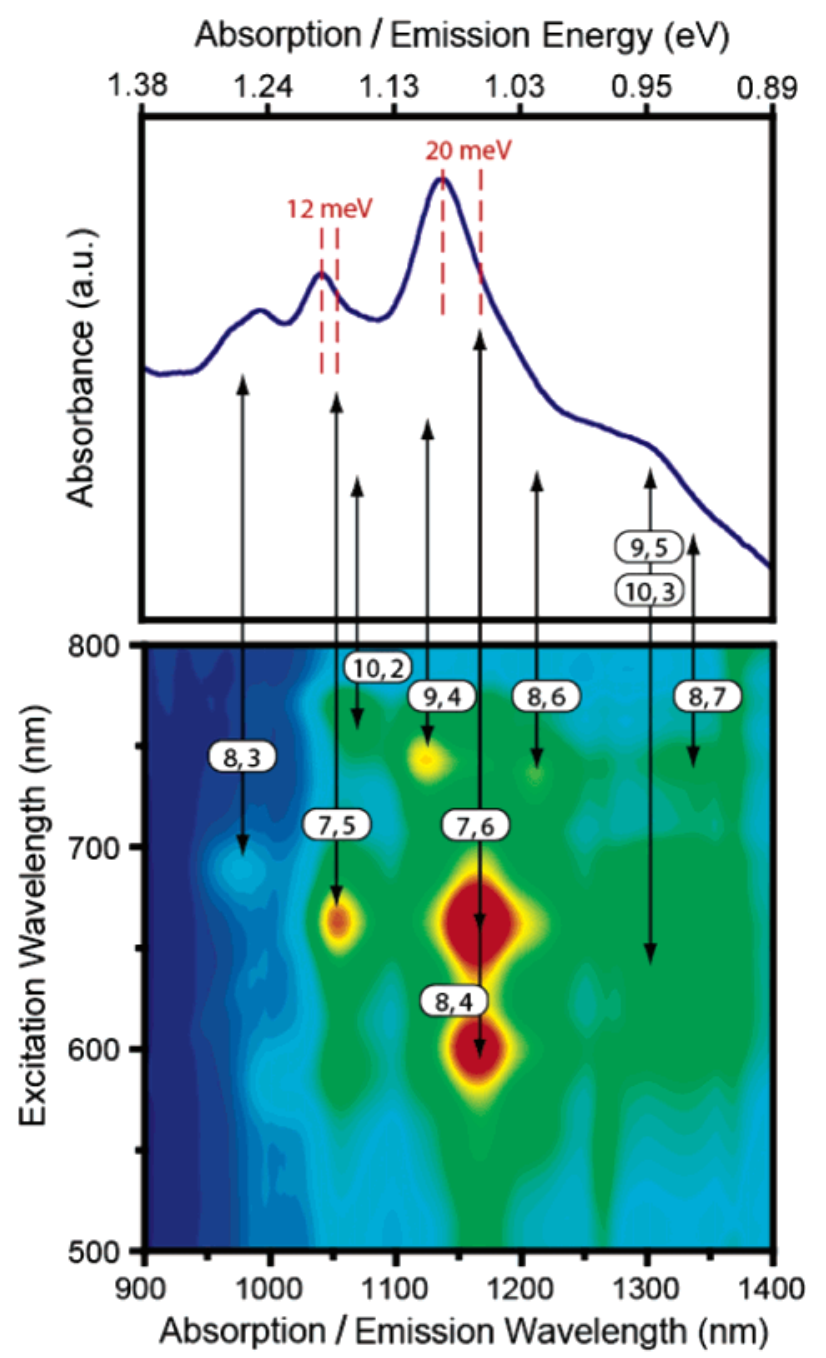

Figure 5. Overlay of the optical absorption and fluorescence data for the unbuffered SWNT-PAA composite film. The prominent SWNT peak positions are labeled on the fluorescence map on the basis of ref 3 and correlated to the absorption spectrum to show the apparent Stokes shifts.

shift is due to interactions between the SWNTs, or the SWNTs with the PAA, in the composite. Finally, conventional wisdom would suggest that the features in all of these spectra are sufficiently resolved to indicate good dispersion. ${ }^{10}$ However, the changes in the peak structure leading to a broader and redshifted absorption in the buffered sample compared to the unbuffered one (particularly at ca. $950 \mathrm{~nm}$ ) supports the SANS results that enhanced dispersion is achieved for the unbuffered composite.

Fluorescence spectroscopy is reported as a sensitive measure of SWNT dispersion, since SWNT bundling quenches the NIR fluorescence associated with the narrow band gap of the semiconducting SWNT species. ${ }^{11-14}$ A comparison of the fluorescence maps for the unbuffered and buffered PAA composites, shown in Figure 4, illustrates a pronounced difference in their spectral emissions. The peak assignments are consistent with previous work using sodium dodecyl sulfate (SDS) $-\mathrm{D}_{2} \mathrm{O}$ dispersions ${ }^{14,25}$ although the measured positions exhibit about $50 \mathrm{meV}$ red-shifts. The fluorescence intensity of the peak maximum for the most prominent $(7,6)$ SWNT was calculated to be 2.5 times higher in the unbuffered sample compared to the buffered composite. In addition, the Lorentzianfitted full width at half maximum (fwhm) was $54 \mathrm{meV}$ compared to $65 \mathrm{meV}$ for the buffered sample.

The higher peak intensity and narrower fluorescence emission in the unbuffered composite is attributed to an improved dispersion due to debundled SWNTs, ${ }^{12}$ suggesting that the lower $\mathrm{pH}$ acts in the PAA composite to maintain the individual dispersion of the tubes from the precursor solution. Such a finding is consistent with the SANS data; however, it is opposite of previous work on solution-phase samples of SDS-dispersed SWNTs where acidification significantly quenched the fluorescence intensity below $\mathrm{pH} 3.2 .{ }^{12}$ Therefore, the fluorescence data in Figure 4 suggests that $\mathrm{pH}$ affects the interactions between ssDNA-wrapped SWNTs and the PAA polymer during composite preparation. Further evidence for this claim is derived from redissolving the SWNT-PAA composite films in $\mathrm{D}_{2} \mathrm{O}$ and acquiring equivalent fluorescent maps (data not shown). For these redissolved samples, the SWNT peak positions were comparable between buffered and unbuffered samples; however, the fluorescence intensity was about $33 \%$ higher in the buffered case, consistent with the previous solution-phase analysis in SDS-dispersed SWNT solutions. ${ }^{12}$ These fluorescence results for the SWNT-PAA composites imply that the significant quenching effects of reduced $\mathrm{pH}$ are less important for the SWNTs upon composite film casting than the effects of the polymer's interactions with the SWNTs. Thus, the factors influencing the measured composite fluorescence may derive from selective interactions between the protonated PAA and ssDNA-wrapped SWNTs, including the possibility for $\mathrm{pH}$-driven changes in the conformation of the polymer that lead to exclusion and aggregation of the SWNTs, ${ }^{26}$ or from a change
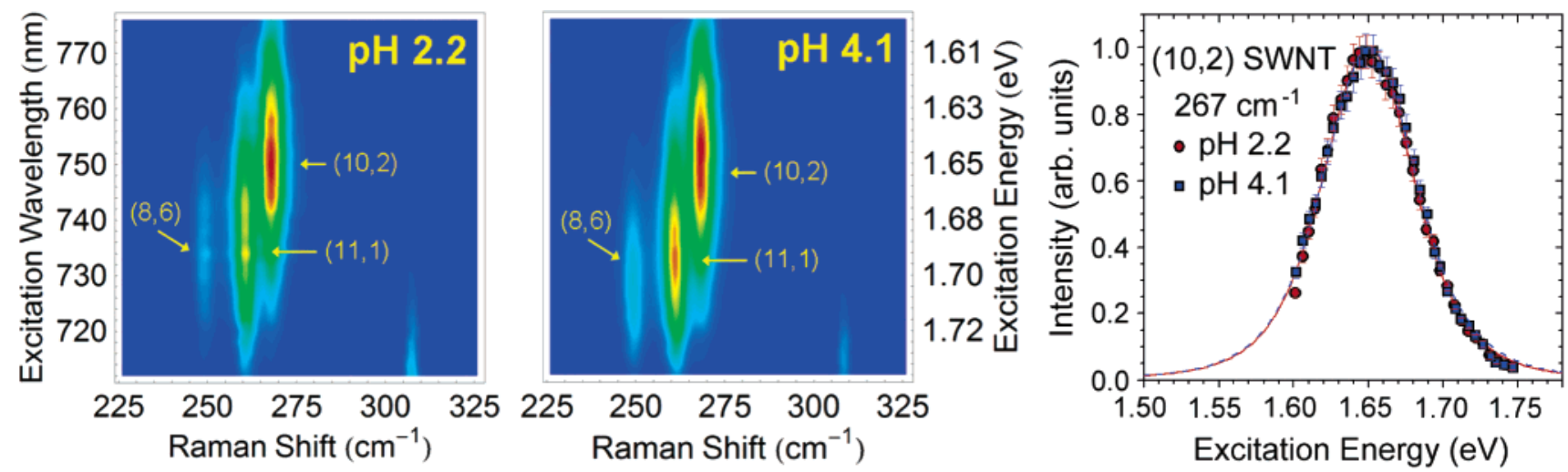

Figure 6. Radial breathing mode resonant Raman scattering for the SWNT composites, with the chirality of specific SWNT type indicated. The plot to the right shows a comparison of the $267.8 \mathrm{~cm}^{-1}$ line shape for $(10,2)$ SWNTs versus excitation energy, where the curves are Lorentzian fits through the data. For the squared Lorentzian fit shown, ${ }^{18}$ the fwhm values are, respectively, 73 and $75 \mathrm{meV}$ for the $\mathrm{pH} 2.2$ and pH 4.1 composites, comparable to the approximately $65 \mathrm{meV}$ value extracted from ref 18 for SWNTs in SDS. 
in the balance of interactions allowing an entropic PAAmediated depletion attraction ${ }^{5}$ to aggregate adjacent SWNTs during solvent evaporation.

An overlay of the optical absorption data with the fluorescence map for the unbuffered composite sample, shown in Figure 5, provides SWNT chirality assignments and evaluation of any Stokes shifts. The predominant peaks in the optical absorption spectrum represent a convolution of the SWNT chiralities present as observed by the fluorescence map. The measured red-shift in fluorescence peak positions are consistent with previous reports. ${ }^{12,14}$ The present Stokes shift values for the SWNT-PAA composites are slightly higher than values calculated for specific chiralities (ca. 5-7 meV) in $\mathrm{SDS}-\mathrm{D}_{2} \mathrm{O}$ dispersions. ${ }^{14}$ However, the effects of the dispersing media and SWNT properties (including purity, chirality distribution, etc.) are expected to dramatically influence the degree of Stokes shifts.

Also measured was the resonant Raman Stokes scattering obtained for the two composites. Focus is on the RBM; no notable difference was measured in the $\mathrm{D}$ and $\mathrm{G}$ band scattering (not shown). In Figure 6, each peak in the plane of excitation energy and Raman frequency corresponds to a specific SWNT diameter. In this plot, Raman intensity has been normalized to account for fluctuations in excitation laser power, and SWNT chirality assignments have been made on the basis of work previously published ${ }^{15-19}$ by other groups. The intensity plots are nearly identical in appearance for the two samples. To scrutinize any difference, we focus on a comparison of the line shape for the $267.8 \pm 0.5 \mathrm{~cm}^{-1}$ peak for $(10,2)$ SWNTs versus the excitation energy, as shown in the lower panel of Figure 6. The data have been normalized to the same peak intensity to facilitate comparison and smoothed through one cycle of nearestneighbor averaging. The fwhm from the Lorentzian fits are 73 and $75 \mathrm{meV}$ for $\mathrm{pH}=2.2$ and 4.1 , respectively, which is nearly equivalent to the measured values from NIR fluorescence mapping. In general, the results show that both samples are consistent with what is reported in the literature for SDSdispersed SWNTs in solution. ${ }^{15-19}$ We note that, by the criterion of O'Connell et al., ${ }^{15}$ both composite samples are debundled. This suggests both that the induced variation in dispersion can be specifically categorized as the diffuse clustering or overlapping aggregation of individual, nonbundled SWNTs, and that the apparent absence of bundles is not sufficient to allow for claims of individualized dispersion on the basis solely of Raman spectroscopy.

\section{Conclusions}

In conclusion, model polymer composites of ssDNAstabilized SWNTs dispersed in PAA are used for a systematic evaluation of SANS and the three spectroscopic metrologies currently used to assess SWNT dispersion in suspensions and composites. By varying the $\mathrm{pH}$ of the precursor PAA-SWNT solution, we demonstrate a controlled change in the resulting dispersion. The more dispersed composite was shown to be "nanodispersed" as individual SWNTs while the least dispersed consisted of diffuse clusters of individual SWNTs. From this comparison, we have evaluated the effectiveness of the various methods in terms of their sensitivity to SWNT clustering. SANS provides the most direct measure of nanotube dispersion, followed by NIR fluorescence spectroscopy. Resonant Raman scattering and optical absorption spectroscopy are useful, but the limits of these techniques for assessing the relative level of dispersion need to be clearly understood, as they provide measures with more subtle changes in signal. As such, they should be limited to homogeneous samples (in the case of transmission measurements) or as a means of detecting SWNT bundling (in the case of resonant Raman scattering). On the basis of our results, the independent use of any of these techniques can be improved in its interpretation, which should increase the ability to distinguish true SWNT dispersion from other less desirable scenarios. A truly rigorous measure of SWNT dispersion, however, would clearly be best made though a combination of all four of these metrologies.

Acknowledgment. We acknowledge the support of the National Institute of Standards and Technology in providing the neutron research facilities used in this work. The NanoPower Research Laboratories acknowledges financial support from BP Solar; NASA: Grant Nos. NAG3-2828, NCC3-956, NNC05GA14G; and the Department of Defense. J.A.F., I.M., and J.R.S. also acknowledge the support of a National Research Council postdoctoral fellowship.

\section{References and Notes}

(1) See, for example, Baughman, R. H.; Zakhidov, A. A.; de Heer, W. A. Science 2002, 297, 787.

(2) Schaefer, D.; Brown, J. M.; Anderson, D. P.; Zhao, J.; Chokalingam, K.; Tomlin, D.; Ilavsky, J. J. Appl. Crystallogr. 2003, 36, 553.

(3) Schaefer, D. W.; Zhao, J.; Brown, J. M.; Anderson, D. P.; Tomlin, D. W. Chem. Phys. Lett. 2003, 375, 369.

(4) Rols, S.; Anglaret, E.; Sauvajol, J. L.; Coddens, G.; Dianoux, A. J. Appl. Phys. A-Mater. Sci. Process. 1999, 69, 591.

(5) Wang, H.; Zhou, W.; Ho, D. L.; Winey, K. I.; Fischer, J. E.; Glinka, C. J.; Hobbie, E. K. Nano Lett. 2004, 4, 1789.

(6) Yurekli, K.; Mitchell, C. A.; Krishnamoorti, R. J. Am. Chem. Soc. 2004, 126, 9902.

(7) Zhou, W.; Islam, M. F.; Wang, H.; Ho, D. L.; Yodh, A. G.; Winey, K. I.; Fischer, J. E. Chem. Phys. Lett. 2004, 384, 185.

(8) Hough, L. A.; Islam, M. F.; Hammouda, B.; Yodh, A. G.; Heiney, P. A. Nano Lett. 2006, 6, 313.

(9) Bauer, B. J.; Hobbie, E. K.; Becker, M. L. Macromolecules 2006 , $39,2637$.

(10) Landi, B. J.; Ruf, H. J.; Worman, J. J.; Raffaelle, R. P. J. Phys. Chem. B 2004, 108, 17089 .

(11) Bachilo, S. M.; Strano, M. S.; Kittrell, C.; Hauge, R. H.; Smalley, R. E.; Weisman, R. B. Science 2002, 298, 2361.

(12) O'Connell, M. J.; et al. Science 2002, 297, 593

(13) Maeda, Y.; et al. J. Phys. Chem. B 2004, 108, 18395.

(14) Jones, M.; Engtrakul, C.; Metzger, W. K.; Ellingson, R. J.; Nozik, A. J.; Heben, M. J.; Rumbles, G. Phys. Rev. B 2005, 71, 115426.

(15) O'Connell, M. J.; Sivaram, S.; Doorn, S. K. Phys. Rev. B 2004, 69,235415

(16) Ericson, L. M.; Pehrsson, P. E. J. Phys. Chem. B 2005, 109, 20276.

(17) Heller, D. A.; Barone, P. W.; Swanson, J. P.; Mayrhofer, R. M.; Strano, M. S. J. Phys. Chem. B 2004, 108, 6905.

(18) Fantini, C.; Jorio, A.; Souza, M.; Strano, M. S.; Dresselhaus, M. S.; Pimenta, M. A. Phys. Rev. Lett. 2004, 93, 147406.

(19) Doorn, S. K.; Heller, D. A.; Barone, P. W.; Usrey, M. L.; Strano, M. S. Appl. Phys. A: Mater. Sci. Process. 2004, 78, 1147.

(20) Zheng, M.; Jagota, A.; Semke, E. D.; Diner, B. A.; McLean, R. S.; Lustig, S. R.; Richardson, R.E.; Tassi, N. G. Nat. Mater. 2003, 338-341.

(21) Certain equipment or materials are identified in this paper in order to specify the experimental procedure adequately. Such identification is not intended to imply endorsement by the National Institute of Standards and Technology, nor is it intended to imply that the materials or equipment identified are necessarily the best available.

(22) Bauer, B. J.; Becker, M. L.; Bajpai, V.; Fagan, J. A.; Hobbie, E. K.; Migler, K.; Guttman, C. M.; Blair, W. R. Macromolecules, submitted 2006.

(23) Mandelbaum, I.; Bolshtyansky, M.; Heinz, T. F.; Walker, A. R. H. J. Opt. Soc. Am. B 2006, 23, 621-627.

(24) Arepalle, S.; Nikolaev, P.; Gorelik, O.; Hadjiev, V. G.; Holmes, W.; Files, B.; Yowell, L. Carbon 2004, 42, 1783-1791.

(25) Weisman, R. B.; Bachilo, S. M. Nano Lett. 2003, 3, 1235.

(26) Grunlan, J. C.; Liu, L.; Kim, Y. S. Nano Lett. 2006, 6, 911-915. 